\title{
基于 $l_{k}$ 范数正则化的 SAR 复图像快速自适应去噪 方法
}

\author{
王卫威 (1) , 王正明(2), 袁震宇(2), 李明山 ${ }^{1}$ \\ (1) 国防科技大学信息系统与管理学院, 长沙 410073; \\ (2) 国防科技大学理学院, 长沙 410073 \\ *通信作者 E-mail: superbcwang@hotmail.com
}

收稿日期: 2008-04-19; 接受日期: 2009-01-24

国家自然科学基金（批准号: 60572136)、国防科技大学预研基金（批准号: JC07020005）资助项目

\begin{tabular}{|c|c|}
\hline $\begin{array}{l}\text { 摘要 文中从参数估计角度研究基于 } l_{k} \text { 范数正则化的 SAR 复图像域快速自适应 } \\
\text { 去噪方法. 首先利用凸半二次正则化思想建立去噪模型与病态逆问题之间的联系, 并 } \\
\text { 依据迭代表达式进行参数估计方差与有偏 CRB 的比较, 从而分析得到现有参数选择 } \\
\text { 方法理论上的不足. 然后对模型求解迭代表达式进行分析, 得到包含正则化参数的模 } \\
\text { 型解. 继而应用最小化均方误差与单调有界数列原理, 得到正则参数的选择方法与模 } \\
\text { 型解的解析表达式, 避免了求解的迭代过程, 可以快速、自适应地实现去噪处理, 并从 } \\
\text { 理论上分析得到计算量减少的具体数值. 最后研究了去噪模型对点目标分辨率的影响, } \\
\text { 建立了正则参数与分辨率的关系. 仿真与实测 SAR 图像去噪结果验证了结论的正确 } \\
\text { 性与方法的有效性. }\end{array}$ & $\begin{array}{l}\text { 关键词 } \\
\mathrm{SAR} \text { 复图像 } \\
\text { 去噪 } \\
l_{k} \text { 范数正则化 } \\
\text { 参数选择 } \\
\text { 模型求解 } \\
\text { 自适应 }\end{array}$ \\
\hline
\end{tabular}

\section{0 引言}

合成孔径雷达 (SAR) 图像在目标检测 [1] 与识 别、军事监测等方面得到广泛应用. SAR 图像质量 越高, 其后续应用效能越好 ${ }^{[2]}$. 通过对成像后的图像 数据运用数学理论进行处理来提高 SAR 图像质量, 成本低且效果好 ${ }^{[3]}$. SAR 图像去噪是其中一个重要 的研究课题.

SAR 图像去噪可以看作病态逆问题, 利用正则 化方法求解. Cetin 等 ${ }^{[4]}$ 提出了一种基于 $l_{k}$ 范数 正则化的 SAR 图像增强方法, 将增强问题转化为具 有约束的优化问题. 但其模型中的降质算子较为理 想化, 且难以应用到除聚束式以外的 SAR 成像系统. 令其为单位阵, 可实现去噪功能. 文献 $[5,6]$ 将其模 型与方法扩展到复图像域及功率图像域.

目前, 基于 $l_{k}$ 范数正则化 SAR 图像去噪模型求
解在速度和自动化程度上均需要进行改进, 表现为模 型求解需要进行多次迭代, 模型参数较难自动确定. 不管模型设计与参数选择, 目的均是达到图像处理的 最优效果. 因此, 图像处理可看作基于图像最优性能 的优化问题. 作为去噪处理不可缺少的一个环节, 对 去噪的性能分析与评估可以为模型与求解算法的设 计提供反馈信息, 可通过两种方式来进行. 一是利用 SAR 图像质量评价指标进行对比; 二是分析去噪模 型的最优性能作为标准对处理性能进行对比、预测. 本文将去噪问题看作从有限含噪观测数据中对目标 参数的高精度估计过程, 对其性能的分析就转化为 对参数估计精度的分析. 针对基于 $l_{k}$ 范数正则化的 SAR 复数据域正则化去噪模型, 通过得到模型解的 解析表达式, 利用统计量的最优性能分析得到精确的 模型参数确定方法, 避免了耗时的迭代过程, 计算速 度快, 自动化程度高, 并在仿真与实测 SAR 图像中 
均取得了理想的去噪效果.

\section{1 问题描述}

\subsection{SAR 图像正则化去噪模型与迭代求解}

一般情况下, 在 SAR 复图像域, 观测模型具有 如下形式 ${ }^{[4,6]}$ :

$$
g=f+\varepsilon
$$

其中, $\boldsymbol{g}$ 为含噪观测信号, $\boldsymbol{f}$ 为真实信号, $\boldsymbol{\varepsilon}$ 为 Gauss 白噪声, 均为 $M N \times 1$ 的列向量, $M, N$ 分别表示图 像的行数与列数 (事实上, 根据后面的分析, 求解过 程可在图像数据的二维矩阵情况下进行). 假定噪声 独立同分布且 $\boldsymbol{\varepsilon} \sim \mathrm{CN}\left(0, \sigma_{g}^{2} \boldsymbol{I}_{M N}\right)$. 对于 $\mathrm{SAR}$ 幅度 与功率图像, 噪声是乘性的, 需要进行对数变换将观 测模型变为加法模型 ${ }^{[7]}$. 本文中仅研究复图像域情 况, 但其方法可扩展到幅度与功率图像处理中.

由于随机噪声的影响, 去噪模型求解存在不稳 定性, 为数学意义上的病态问题. 为改善病态性, 需 要挖掘 SAR 图像的相关先验信息构建正则项进行求 解. 而大多数 SAR 图像具有稀疏性质 ${ }^{[8]}$, 故正则项 可利用具有稀疏表示能力的 $l_{k}$ 范数来构建:

$$
R(\boldsymbol{f})=\|\boldsymbol{f}\|_{k}^{k},
$$

其中, $k$ 为正实数, $0<k<2$. 该约束项与数据拟合 项构成如下正则化去噪模型 ${ }^{[4 \sim 6]}$ :

$$
\hat{\boldsymbol{f}}=\underset{\boldsymbol{f}}{\arg \min }\|\boldsymbol{g}-\boldsymbol{f}\|_{2}^{2}+\lambda\|\boldsymbol{f}\|_{k}^{k},
$$

或记为如下具有约束的优化问题:

$$
\left\{\begin{array}{l}
\min \|\boldsymbol{g}-\boldsymbol{f}\|_{2}^{2} \\
\|\boldsymbol{f}\|_{k}^{k}=\eta
\end{array}\right.
$$

对应的目标函数为

$$
J(\boldsymbol{f})=\|\boldsymbol{g}-\boldsymbol{f}\|_{2}^{2}+\lambda\|\boldsymbol{f}\|_{k}^{k},
$$

$\lambda(\lambda>0)$ 为正则化参数, $\eta$ 与 $\lambda$ 有关.

为避免 $R(\boldsymbol{f})=\|\boldsymbol{f}\|_{k}^{k}$ 在零点邻域位置不可微, 做如下平滑近似 ${ }^{[4]}$ :

$$
\|\boldsymbol{f}\|_{k}^{k} \approx \sum_{i=1}^{M N}\left(\left|f_{i}\right|^{2}+\mu\right)^{\frac{k}{2}}
$$

$\mu$ 为较小正实数, 可取为 $10^{-5}$. 那么, 目标函数 (5)
可写为

$$
J(\boldsymbol{f})=\|\boldsymbol{g}-\boldsymbol{f}\|_{2}^{2}+\lambda \sum_{i=1}^{M N}\left(\left|f_{i}\right|^{2}+\mu\right)^{\frac{k}{2}} \triangleq \sum_{i=1}^{M N} J\left(f_{i}\right) .
$$

易知, $J\left(f_{i}\right)$ 具有与 $J(\boldsymbol{f})$ 相同的性质, 因此只需对 $J\left(f_{i}\right)$ 进行研究.

模型 (3) 需要利用数值方法迭代求解. 对目标函 数 (7) 应用复信号的求导方法 ${ }^{[9]}$ 关于 $f_{i}$ 进行求导, 且令其一阶导数为 0 , 可得稳定点方程:

$$
-\left(g_{i}-f_{i}\right)^{*}+\frac{\lambda k}{2}\left(\left|f_{i}\right|^{2}+\mu\right)^{\frac{k}{2}-1} f_{i}^{*}=0,
$$

上标 $*$ 表示共轭. 相应的迭代过程可为

$$
\hat{f}_{i}^{(n+1)}=g_{i} / \boldsymbol{H}_{i i}^{(n)},
$$

上标 $(n)$ 表示第 $n$ 次迭代. $\boldsymbol{H}_{i i}=1+\frac{\lambda k}{2}\left(\left|f_{i}\right|^{2}+\right.$ $\mu)^{\frac{k}{2}-1}$.

\section{2 现有正则参数选择方法分析}

为达到最优处理效果, 需要正确确定该模型中 的正则参数 $\lambda$. 现有的参数确定方法有 $\mathrm{L}$ 曲线、广 义交叉校验 $(\mathrm{GCV})$ 、离差原理等, 但这些方法大都应 用于 Tikhonov 正则化, 且较少从理论上得到明确的 解析表达式. 文献 [6] 将模型 (3) 的求解与广义岭估 计联系起来, 得到正则化参数的选择方法 $\lambda k=2 \sigma_{g}^{2}$. 但这种联系仅建立在模型迭代求解式 (9) 与广义岭 估计解形式相同基础上, 并没有从理论上进行分析, 且仍需要迭代, 不可避免地存在误差, 并且耗时较 长. 以下从去噪模型与病态逆问题、参数估计方差 与 Cramer-Rao 下界 (CRB) 比较两方面来分析该方 法理论上的不足.

文献 [10] 证明了 Tikhonov 正则化与岭估计之 间的统一性. 为利用其证明过程, 引入凸半二次正则 化方法 ${ }^{[11]}$. 该方法可以引入一个新的和原目标函数 有相同极值点的凸目标函数, 但引入的新目标函数更 易于数值处理. 令

$$
\varphi\left(\left|f_{i}\right|\right) \triangleq\left(\left|f_{i}\right|^{2}+\mu\right)^{k / 2},
$$

那么存在一个严格凸且降的函数 $\psi$, 使得

$$
\varphi\left(\left|f_{i}\right|\right)=\inf _{0<w_{i} \leqslant \infty}\left(w_{i}\left|f_{i}\right|^{2}+\psi\left(w_{i}\right)\right) .
$$

且对于每个固定的 $\left|f_{i}\right|$, 函数 $w_{i}\left|f_{i}\right|^{2}+\psi\left(w_{i}\right)$ 有唯一 的极小值点 $w_{i}$ : 


$$
w_{i}=\frac{\phi^{\prime}\left(\left|f_{i}\right|\right)}{2\left|f_{i}\right|}=\frac{k}{2}\left(\left|f_{i}\right|^{2}+\mu\right)^{\frac{k}{2}-1} .
$$

通过计算构造 $\psi\left(w_{i}\right)$ 如下:

$$
\psi\left(w_{i}\right)=\left[\left(\frac{2}{k}\right)^{\frac{k}{k-2}}-\left(\frac{2}{k}\right)^{\frac{2}{k-2}}\right] w_{i}^{\frac{k}{k-2}}+w_{i} \mu .
$$

令 $\boldsymbol{w}=\operatorname{diag}\left(w_{i}\right)$ 为 $M N \times M N$ 的对角阵, 由于

$$
\begin{aligned}
\left\|\boldsymbol{w}^{1 / 2} \boldsymbol{f}\right\|_{2}^{2} & =\sum_{i=1}^{M N} w_{i}\left|f_{i}\right|^{2} \\
& =\frac{k}{2} \sum_{i=1}^{M N}\left(\left|f_{i}\right|^{2}+\mu\right)^{\frac{k}{2}}=\frac{k}{2}\|\boldsymbol{f}\|_{k}^{k} .
\end{aligned}
$$

需要说明的是, 由于 $\mu$ 的作用在于保持可微性, 在必 要时可以按照可微与否进行添加与删减.

根据以上分析, 原图像去噪模型的目标函数最小 化可写为

$$
\min \left\|\left(\begin{array}{c}
\mathbf{I} \\
\sqrt{\frac{2 \lambda}{k}} \boldsymbol{w}^{\frac{1}{2}}
\end{array}\right) \boldsymbol{f}-\left(\begin{array}{c}
\boldsymbol{g} \\
\mathbf{0}
\end{array}\right)\right\|_{2}^{2} .
$$

其中, $\mathbf{I}$ 为 $M N \times M N$ 的单位阵. 令 $\boldsymbol{A}=$ (I $\left.\sqrt{2 \lambda / k} \boldsymbol{w}^{1 / 2}\right)^{\mathrm{T}}$ 为 $2 M N \times M N$ 的列满秩矩阵, $\boldsymbol{B}=\left(\begin{array}{ll}\boldsymbol{g} & \mathbf{0}\end{array}\right)^{\mathrm{T}}$ 为 $2 M N \times 1$ 的列向量. 因此, 模型 (3) 可以看作求解 $\min \|\boldsymbol{A f}-\boldsymbol{B}\|_{2}^{2}$ 问题. 可以看到, 与一 般的线性方程不同的是, $\boldsymbol{A}$ 为 $\boldsymbol{f}$ 的函数, 即 (14) 式 为非线性病态问题. 这与广义岭估计所依赖的线性 病态逆问题并不一致. 所以文献 [6] 以迭代表达式形 式与广义岭估计相似, 而将两者联系起来, 并不严谨.

此外, 评估参数估计性能的重要指标就是参数估 计的偏差、协方差、均方误差 (MSE) 等统计量. 广义 岭估计的最优参数选择建立在最小化 MSE 基础上, 其中涉及到方差的计算. 衡量参数估计性能的一个 主要指标就是参数估计值的 (协) 方差与其下界的偏 离程度, 较为常用的是 CRB. 正则化方法需要达到偏 差与方差的折中, 一般情况下为有偏估计. 下面利用 方差与有偏 $\mathrm{CRB}$ 的比较来分析文献 [6] 方法的不足.

根据迭代表达式 (9), 可假定估计值为

$$
\hat{f}_{i}=\left(1+\frac{\lambda k}{2}\left(\left|f_{i}\right|^{2}+\mu\right)^{\frac{k}{2}-1}\right)^{-1} g_{i} .
$$

估计偏差 $b\left(f_{i}\right)$ 为

$$
b\left(f_{i}\right)=\frac{-\lambda k\left(\left|f_{i}\right|^{2}+\mu\right)^{\frac{k}{2}-1}}{2+\lambda k\left(\left|f_{i}\right|^{2}+\mu\right)^{\frac{k}{2}-1}} f_{i} .
$$

估计值方差为

$$
\operatorname{Var}\left(\hat{f}_{i}\right)=\left(1+\frac{\lambda k}{2}\left(\left|f_{i}\right|^{2}+\mu\right)^{\frac{k}{2}-1}\right)^{-2} \sigma_{g}^{2} .
$$

因此, 该估计过程为有偏估计, 而且偏差小于 0 . 这使 得处理后图像像素值变小, 比原图像变暗. 而且偏差 绝对值为 $\lambda k$ 的增函数, 方差为 $\lambda k$ 的减函数. 可知, 估计值偏差与方差不可能同时达到最小.

由于模型 (3) 可看作具有约束的参数估计问题, 而文献 [12] 得到了具有约束的参数估计边界, 可依 据相关结论对方差与 $\mathrm{CRB}$ 进行比较.

定理 ${ }^{[12]}$ 令观测数据为 $\boldsymbol{X}$, 待估参数为 $\boldsymbol{\theta}, \boldsymbol{X}$ 关于 $\boldsymbol{\theta}$ 的条件分布表示为 $p_{\boldsymbol{\theta}}$. 假设满足正则条 件, 参数空间 $\Theta_{C} \subset \mathrm{R}^{n}$ 由等式 $G_{\boldsymbol{\theta}}=0$ 与不等

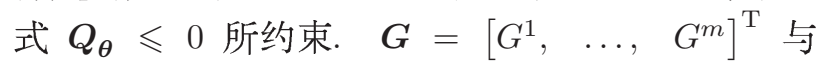
$\boldsymbol{Q}=\left[\begin{array}{lll}Q^{1}, & \ldots, Q^{n}\end{array}\right]^{\mathrm{T}}$ 连续可微. 假定 $p \times n$ 梯度 矩阵 $\nabla \boldsymbol{G}_{\boldsymbol{\theta}}$ 秩为 $r, r \leqslant m$, 那么对于任意具有均值为 $m_{\theta}$ 的估计值 $\theta$, 估计值的协方差矩阵 $\Sigma_{\theta}$ 满足矩阵 不等式:

$$
\boldsymbol{\Sigma}_{\boldsymbol{\theta}} \geqslant \boldsymbol{B}_{c}
$$

其中, $\boldsymbol{B}_{c}=\left[\nabla \boldsymbol{m}_{\boldsymbol{\theta}}\right] \boldsymbol{Q}_{\boldsymbol{\theta}} \boldsymbol{J}_{\boldsymbol{\theta}}^{-1}\left[\nabla \boldsymbol{m}_{\boldsymbol{\theta}}\right]^{\mathrm{T}}, \boldsymbol{Q}_{\boldsymbol{\theta}}$ 为 $n \times n$ 的 幂等、秩为 $p-r$ 的矩阵:

$$
\boldsymbol{Q}_{\boldsymbol{\theta}}=\left\{\begin{array}{l}
\mathbf{I}, \text { 如果 } \boldsymbol{\theta} \text { 是 } \boldsymbol{\Theta}_{c} \text { 的正则点; } \\
\mathbf{I}-\boldsymbol{J}_{\boldsymbol{\theta}}^{-1}\left[\nabla \boldsymbol{G}_{\boldsymbol{\theta}}\right]^{T}\left\{\left[\nabla \boldsymbol{G}_{\boldsymbol{\theta}}\right] \boldsymbol{J}_{\boldsymbol{\theta}}^{-1}\left[\nabla \boldsymbol{G}_{\boldsymbol{\theta}}\right]^{\mathrm{T}}\right\}^{\dagger} \\
{\left[\nabla \boldsymbol{G}_{\boldsymbol{\theta}}\right], \text { 其他, }}
\end{array}\right.
$$

其中, $\boldsymbol{J}_{\boldsymbol{\theta}}$ 为无约束条件下的 Fisher 信息阵.

虽然该定理建立在实数域的推导基础上, 但在模 型 (4) 中, 等式约束条件

$$
G=\sum_{i=1}^{M N}\left(\left|f_{i}\right|^{2}+\mu\right)^{\frac{k}{2}}-\eta=0
$$

为复变量的实函数, 因此该定理对于模型 (4) 是适用 的. 通过简单计算, 并利用不等式

$$
\sum_{i=1}^{M N}\left(\left|f_{i}\right|^{2}+\mu\right)^{k-1} \gg\left(\left|f_{i}\right|\left|f_{j}\right|+\mu\right)^{\frac{k-1}{2}}
$$

可得估计值的有偏 CRLB 为

$$
\boldsymbol{B}_{c}=\sigma_{g}^{2} \operatorname{diag}(\boldsymbol{A}),
$$


其中,

$$
\begin{aligned}
A_{i i}= & {\left[4+\lambda k(4-k)\left(\left|f_{i}\right|^{2}+\mu\right)^{\frac{k}{2}-1}\right]^{2} } \\
& \cdot\left[2+\lambda k\left(\left|f_{i}\right|^{2}+\mu\right)^{\frac{k}{2}-1}\right]^{-4} .
\end{aligned}
$$

根据 (17) 和 (18) 式, 有

$$
\sigma_{g}^{2} A_{i} \leqslant\left(1+\frac{\lambda k}{2}\left(\left|f_{i}\right|^{2}+\mu\right)^{\frac{k}{2}-1}\right)^{-2} \sigma_{g}^{2} .
$$

可解得 $k \geqslant 2$. 当 $k=2$ 时方差达到 CRB. 但该条件 不符合稀疏性条件.

分析其计算错误的原因在于, 在计算过程中, $\hat{f}$ 需要进行迭代求解, 而上面仅仅简单的利用了迭代 表达式, 过于理想化, 并不能替代最终迭代求解结果. 而且, 岭估计与广义岭估计的最优参数以及各种边界 均是从参数的最终解析解来进行分析的, 而文献 [6] 的逻辑顺序则与之相反. 下面通过分析迭代求解过 程得到模型 (3) 的解析解.

\section{2 迭代解的解析表达式分析}

文献 [6] 证明了目标函数 (5) 具有唯一极小值, 而且可以通过上面的迭代过程 (14) 收敛到该极小值. 下面仅对 $f_{i}$ 进行分析. 令初值为 $\hat{f}_{i}^{(0)}=g_{i}$. 为表达 简洁, 在不引起混淆的情况下, 省略下标 $i$. 由迭代过 程产生的序列 $\left\{\hat{f}^{(1)}, \hat{f}^{(2)}, \ldots, \hat{f}^{(n)}, \ldots\right\}$ 为复数列. 复 数列收敛的必要充分条件为由各项的实部与虚部构 成的两个实数列都收玫, 可以验证该序列实部与虚部 单调、有界, 且界为 0 . 根据单调有界原理可知序列 极限存在. 令序列极限为 $x$, 对 (15) 式两边取极限得

$$
2\left(|x|^{2}+\mu\right)^{1-\frac{k}{2}} x-2 g\left(|x|^{2}+\mu\right)^{1-\frac{k}{2}}+\lambda k x=0 .
$$

由于上述方程为复数与实数的混合方程, 不便于 分析, 故将 $x$ 分为实部 $x_{R}$ 与虚部 $x_{I}$ 分别进行讨论, 而且在不涉及连续与微分的情况下, 忽略 $\mu$. 此外, 由 于 $k$ 越小, 度量数据向量的结构的稀疏性越合理, 令 $k \rightarrow 0^{+}$. 可得如下方程组

$$
\left\{\begin{array}{l}
2\left(x_{R}^{2}+x_{I}^{2}\right) x_{R}-2 g_{R}\left(x_{R}^{2}+x_{I}^{2}\right)+\lambda k x_{R}=0, \\
2\left(x_{R}^{2}+x_{I}^{2}\right) x_{I}-2 g_{I}\left(x_{R}^{2}+x_{I}^{2}\right)+\lambda k x_{I}=0 .
\end{array}\right.
$$

从单纯方程求解角度, 可得以下解:

(i) $x_{R}=0, x_{I}=0$; (ii) 当 $g_{R}^{2}+g_{I}^{2} \geqslant 2 \lambda k$ 时, 方程存在实数根

$$
x_{I}=\frac{1}{2} g_{I} \pm \frac{1}{2}\left|g_{I}\right| \sqrt{\frac{\left(g_{R}^{2}+g_{I}^{2}\right)-2 \lambda k}{\left(g_{R}^{2}+g_{I}^{2}\right)}} .
$$

迭代过程取初值 $f^{(0)}=g$, 当 $g_{I}>0$ 时,

$$
\begin{aligned}
\hat{f}_{I}^{(1)} & =\left(1+\frac{\lambda k / 2}{\left|\hat{f}^{(0)}\right|^{2}+\mu}\right)^{-1} g_{I} \\
& \geqslant\left(1+\frac{\lambda k / 2}{2 \lambda k}\right)^{-1} g=\frac{4}{5} g_{I} .
\end{aligned}
$$

可推知 $\hat{f}^{(n+1)} \geqslant w_{n} g, w_{n}=\frac{4 w_{n-1}^{2}}{4 w_{n-1}^{2}+1}$, 其中, $w_{0}=1$. 可知 $w_{n}$ 为单调递减数列, 那么同样存在极限, 令其 极限为 $w$, 可得

$$
w=\frac{4 w^{2}}{4 w^{2}+1},
$$

解为 $w=0.5$. 那么,

$$
x_{I} \geqslant \frac{1}{2} g_{I} .
$$

同理, 当 $g_{I}<0$ 时存在 $x_{I} \leqslant g_{I} / 2$. 因此,

$$
x_{I}=\frac{1}{2} g_{I}+\frac{1}{2} g_{I} \sqrt{\frac{\left(g_{R}^{2}+g_{I}^{2}\right)-2 \lambda k}{\left(g_{R}^{2}+g_{I}^{2}\right)}} .
$$

因此, 可以得到如下结论:

(i) 当 $g_{R}^{2}+g_{I}^{2} \geqslant 2 \lambda k$ 时, 估计值收敛到

$$
x=\left(\frac{1}{2}+\sqrt{\frac{\left(g_{R}^{2}+g_{I}^{2}\right)-2 \lambda k}{4\left(g_{R}^{2}+g_{I}^{2}\right)}}\right) g .
$$

(ii) 当 $g_{R}^{2}+g_{I}^{2}<2 \lambda k$ 时, 估计值收玫到 0 .

此外, 可分析收玫的迭代次数. 令 $g_{R}^{2}+g_{I}^{2}=\alpha \lambda k$, $\alpha \geqslant 2, \alpha$ 越大, 收玫速度越慢. 以 $\alpha=2$ 为例进行分 析. 若以 $\left(w_{n}-w_{n-1}\right)^{2} / w_{n}^{2} \leqslant \gamma$ 为迭代终止条件, $\gamma$ 为一极小正实数, 可得

$$
w_{n-1} \leqslant \frac{1}{2} \sqrt{(1+\sqrt{\gamma})^{2}-1}+\frac{1}{2}(1+\sqrt{\gamma}) .
$$

令 $\gamma \leqslant 10^{-6}$, 可知 $w_{n-1} \leqslant 0.5229$. 从图 1 中的 $\left\{w_{n}\right\}$ 序列曲线可以看出, 该曲线可用关于 $n$ 的指数函数 来拟合. 


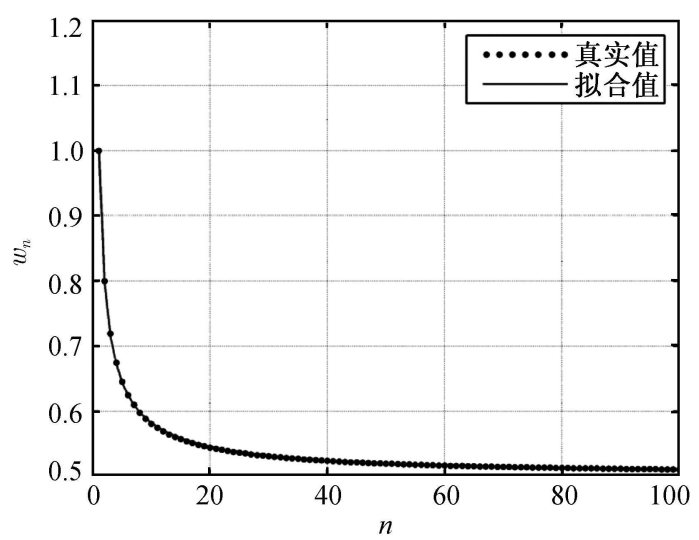

图 1 迭代系数序列拟合曲线

相关拟合表达式为

$$
w_{n}=0.5109 n^{-0.758}+0.4925,
$$

可近似得 $n=42$. 这意味着直接应用解析表达式, 最 乐观情况可以将求解速度提高 42 倍. $\gamma$ 取值越小, 提 高的倍数越大.

分析到此, 得到了 SAR 图像去噪模型的解析解, 该表达式的得出, 可以用于相关理论分析, 而且避免 了迭代求解过程. 可以看到当 $k \rightarrow 0^{+}$时, 单一 $k$ 的 选择对模型解影响不大, 模型解或处理效果取决于 $\lambda k$ 整体的选择. 但是, 此时仍不能达到自动处理的 程度. 下面继续分析参数的选择方法.

\section{3 模型参数确定与求解}

\section{1 模型参数选择方法}

既然可将 SAR 图像去噪性能归结为参数估计性 能, 而且偏差与方差是不可调和的矛盾, 那么利用估 计值的 MSE 进行分析.

令 $2 \lambda k=\beta\left(g_{R}^{2}+g_{I}^{2}\right), \beta \leqslant 1$, 即 $f$ 的估计值 为 $\hat{f}=0.5(1+\sqrt{1-\beta}) g$. 根据均方误差的定义, 对 $\operatorname{MSE}(\hat{f})$ 关于 $\beta$ 求一阶偏导并令其为 0 , 可得

$$
\beta=1-\frac{\left(|f|^{2}-\sigma_{g}^{2}\right)^{2}}{\left(|f|^{2}+\sigma_{g}^{2}\right)^{2}},
$$

可以验证该值可使得 $\operatorname{MSE}(\hat{f})$ 取得最小值. 可以看 到, $\beta$ 的选择与噪声方差、观测数据以及真实数据有 关.

\section{2 模型快速求解方法}

由于 $\beta$ 与真实信号值有关, 而该值是未知的. 虽 然可以利用 Hemmerle-Brantle 等方法 ${ }^{[13]}$, 但仍需要 迭代求解. 文献 [14] 提出广义岭估计的直接解法, 其 核心思想也是单调数列极限原理. 为此, 针对以上分 析结果, 继续应用第 2 节相关过程, 以便能够得到迭 代过程的解析解, 避免迭代过程, 从而提高 SAR 图 像去噪处理速度以及自适应性.

将 $\beta$ 表达式代入 (32) 式, 可得迭代表达式:

$$
\hat{f}^{(n+1)}=\frac{1}{2}\left(1+\frac{\left.|| \hat{f}^{(n)}\right|^{2}-\sigma_{g}^{2} \mid}{\left|\hat{f}^{(n)}\right|^{2}+\sigma_{g}^{2}}\right) g,
$$

初值 $\hat{f}^{(0)}$ 仍为 $\hat{f}^{(0)}=g$. 可以证明迭代数列 $\left\{\hat{f}^{(1)}, \hat{f}^{(2)}, \ldots, \hat{f}^{(n)}, \ldots\right\}$ 仍为单调有界数列, 存在极 限 $x$, 故对上式左右取极限得

$$
x=\frac{1}{2}\left(1+\frac{\left.|| x\right|^{2}-\sigma_{g}^{2} \mid}{|x|^{2}+\sigma_{g}^{2}}\right) g .
$$

下面对 $|x|^{2} \geqslant \sigma_{g}^{2}$ 与 $|x|^{2}<\sigma_{g}^{2}$ 两种情况分别进 行分析.

1) $|x|^{2} \geqslant \sigma_{g}^{2}$.

根据 (34) 式, 构造如下方程组:

$$
\left\{\begin{array}{l}
\left(x_{R}^{2}+x_{I}^{2}\right) x_{R}+\sigma_{g}^{2} x_{R}=\left(x_{R}^{2}+x_{I}^{2}\right) g_{R}, \\
\left(x_{R}^{2}+x_{I}^{2}\right) x_{I}+\sigma_{g}^{2} x_{I}=\left(x_{R}^{2}+x_{I}^{2}\right) g_{I},
\end{array}\right.
$$

可知 $\lambda k=2 \sigma_{g}^{2}$. 虽然与文献 [6] 得到相同的结论, 但 分析过程明显比其在理论上更加严谨、科学. 可以验 证此时 $|x|^{2} \geqslant \sigma_{g}^{2}$.

\section{2) $|x|^{2}<\sigma_{g}^{2}$.}

根据 (34) 式, 且最终迭代结果存在 $x \geqslant 0.5 g$ (见 (27) 式), 则有 $|g|^{2}<4 \sigma_{g}^{2}$, 那么可构造如下方程组:

$$
\left\{\begin{array}{l}
\left(x_{R}^{2}+x_{I}^{2}\right) x_{R}+\sigma_{g}^{2} x_{R}=\sigma_{g}^{2} g_{R}, \\
\left(x_{R}^{2}+x_{I}^{2}\right) x_{I}+\sigma_{g}^{2} x_{I}=\sigma_{g}^{2} g_{I} .
\end{array}\right.
$$

可以看到此时实部与虚部均不为 0 , 可得

$$
x_{I}^{3}+x_{I} \frac{\sigma_{g}^{2} g_{I}^{2}}{g_{R}^{2}+g_{I}^{2}}-\frac{\sigma_{g}^{2} g_{I}^{3}}{g_{R}^{2}+g_{I}^{2}}=0 .
$$

根据 Cardan 公式求解该一元三次方程, 取其实数解 可得 


$$
\begin{aligned}
& x_{I}=\left[\frac{\sigma_{g}^{2} g_{I}^{3}}{2\left(g_{R}^{2}+g_{I}^{2}\right)}\right]^{1 / 3}\left\{1+\left[1+\frac{4 \sigma_{g}^{2}}{27\left(g_{R}^{2}+g_{I}^{2}\right)}\right]^{1 / 2}\right\}^{1 / 3} \\
& +\left[\frac{\sigma_{g}^{2} g_{I}^{3}}{2\left(g_{R}^{2}+g_{I}^{2}\right)}\right]^{1 / 3}\left\{1-\left[1+\frac{4 \sigma_{g}^{2}}{27\left(g_{R}^{2}+g_{I}^{2}\right)}\right]^{1 / 2}\right\}^{1 / 3} .
\end{aligned}
$$$$
\text { 令 } y=\frac{4 \sigma_{g}^{2}}{27\left(g_{R}^{2}+g_{I}^{2}\right)} \text {, 可知 } y \in\left(\frac{1}{27}, \infty\right) \text {. 所以 }
$$

$$
x_{I} \in\left(0,0.5 g_{I}\right) \text {. }
$$

同理可得

$$
x_{R} \in\left(0,0.5 g_{R}\right) .
$$

不满足 $x \geqslant 0.5 \mathrm{~g}$ 条件, 故该情况不成立. 因此, 在该 情况下, $x=0$.

综上所述, 在对 SAR 复图像应用模型 (5) 进行 去噪处理时:

(1) 当 $g_{R}^{2}+g_{I}^{2} \geqslant 4 \sigma_{g}^{2}$ 时, 直接令像素估计值 $\hat{f}$ 为

$$
\hat{f}=\left(\frac{1}{2}+\sqrt{\frac{\left(g_{R}^{2}+g_{I}^{2}\right)-4 \sigma_{g}^{2}}{4\left(g_{R}^{2}+g_{I}^{2}\right)}}\right) g ;
$$

(2) 当 $g_{R}^{2}+g_{I}^{2}<4 \sigma_{g}^{2}$ 时, 令 $\hat{f}=0$.

利用该结论仅需进行 $M N-t$ 个像素点运算, 其 中, $t$ 为满足 $g_{R}^{2}+g_{I}^{2}<4 \sigma_{g}^{2}$ 的像素点个数; 而传统迭 代方法则需进行 $n \times M N$ 个像素点运算, $n$ 为迭代 次数. 由于 SAR 图像的稀疏特性, 减少的计算量是 相当可观的. $\sigma_{g}^{2}$ 可通过对 SAR 复数据的直方图统计 分析来得到.

\section{4 去噪模型对分辨率的影响分析}

提高 SAR 图像质量重要目的之一在于提高图像 分辨率. 最小化 MSE 使得估计估计值 $\hat{f}$ 与真实值 $f$ 之间的差别最小, 达到了理论上的 “最优” 去噪效果. 但模型求解中对提高分辨率作用并不直观. 其中, 一 方面的原因在于模型 (3) 中缺少降质算子 $\boldsymbol{T}$, 另一方 面的原因在于参数选择的原则并没有涉及到分辨率 因素.

目前, 一般利用名义分辨率, 即点目标冲激响应 的 $3 \mathrm{~dB}$ 主瓣宽度来计算 SAR 图像分辨率 ${ }^{[15]}$. 基 于此, 分辨率提高的视觉效果表现为, 中央点目标幅 度值与周围邻近 (像素) 值差别越明显越好, 即主瓣
变窄, 旁瓣值降低. 最极端的情况是理想点冲激响应 (类 $\delta$ 函数). 在本节中, 分析模型 (3) 对 SAR 图像分 辨率的影响.

令 $g_{1}$ 为点目标峰值, $g_{2}$ 为峰值邻近像素值, 很 明显有 $\left|g_{1}\right| \geqslant\left|g_{2}\right|$, 相应的估计值为 $x_{1}, x_{2} . z=\lambda k$, 根据 (29) 式, 有

$$
S=\frac{\left|x_{1}\right|}{\left|x_{2}\right|}=\frac{\left(\left|g_{1}\right|+\sqrt{\left|g_{1}\right|^{2}-2 z}\right)}{\left(\left|g_{2}\right|+\sqrt{\left|g_{2}\right|^{2}-2 z}\right)} .
$$

若要处理后图像分辨率提高, 必须使得 vspace1mm

$$
S>\frac{\left|g_{1}\right|}{\left|g_{2}\right|} \text {. }
$$

根据 (42) 式可得下式:

$S\left(\sqrt{\left|g_{1}\right|^{2}-2 z} \sqrt{\left|g_{2}\right|^{2}-2 z}\right)=-S\left|g_{1}\right|\left|g_{2}\right|+z+S^{2} z$.

继续对方程 (44) 进行求解, 可得

$$
z=-2 \frac{S^{2}\left(\left|g_{1}\right|^{2}+\left|g_{2}\right|^{2}\right)-S\left(S^{2}+1\right)\left|g_{1}\right|\left|g_{2}\right|}{\left(S^{2}-1\right)^{2}} .
$$

由于 $z>0$, 需使得 vspace $1 \mathrm{~mm}$

$$
S>\frac{\left|g_{1}\right|}{\left|g_{2}\right|} \text { 或 } S<\frac{\left|g_{2}\right|}{\left|g_{1}\right|} \text { (不符合条件, 去掉). }
$$

可知, 不管正实数 $z$ 取何值都是可以提高点目标分辨 率的. 因此, 去噪的同时也会提高点目标分辨率. 由于 $S$ 越大, 分辨率越高, 可以说 (45) 式从一定程度上建 立了正则化参数与处理后 SAR 图像点目标分辨率的 关系.

理想情况下, 要求 $\left|x_{1}\right|^{2} /\left|x_{2}\right|^{2} \geqslant 2$, 即 $S \geqslant \sqrt{2}$. 由于 $S(z)$ 为 $z$ 的增函数, 将 $S(z) \geqslant \sqrt{2}$ 代入 (45) 式, 可得

$$
z \geqslant 6 \sqrt{2}\left|g_{1}\right|\left|g_{2}\right|-4\left(\left|g_{1}\right|^{2}+\left|g_{2}\right|^{2}\right) .
$$

因此, 当 $\left|g_{2}\right|^{2} \leqslant 0.5\left|g_{1}\right|$ 时, 不管 $z$ 取何值, 均有 $S \geqslant \sqrt{2}$; 当 $\left|g_{2}\right|^{2}>0.9428\left|g_{1}\right|$ 时, 不管 $z$ 取何值, 均有 $S<\sqrt{2}$.

上面仅是针对理想情况进行分析. 事实上, 在提 高分辨率意义上的最优 $z$ 难以确定. 因为 $z$ 的最大 值为 $0.5|g|$. 对于点目标位置, 要求 $z$ 接近于 0 ; 对于 旁瓣位置, 要求 $z \rightarrow 0.5|g|$. 困难之处在于如何判断 点目标与旁瓣位置. 单纯的降低旁瓣是以减少散射中 心数目 (对于 SAR 图像目标检测的影响则是降低检 
测率, 增加漏警率) 为代价的. SAR 图像中散射中心 的数目是难以准确确定的. 若简单的以统计分布来进 行判断, 则会去除弱散射点目标.

\section{5 实验结果与分析}

在该节中, 利用仿真 SAR 图像与实测 MSTAR 图像进行去噪处理来验证本文方法的有效性与结论 的正确性. 实现软件为 Matlab 6.5.

(1) 仿真复图像.

利用点散射模型 ${ }^{[16]}$ 仿真生成一幅含有四点目 标的 SAR 复图像. 成像参数为: 带宽 $B=0.591 \times 10^{9}$ $\mathrm{Hz}$, 中心频率 $f_{c}=9.599 \times 10^{9} \mathrm{~Hz}($ 与 MSTAR 成 像参数相同), 相位历史大小为 $16 \times 16$, 成像累积角 $\phi_{m}=2.3^{\circ}$, 则其距离向分辨率 $R_{r}=0.254 \mathrm{~m}$, 方位 向分辨率 $R_{c}=0.389 \mathrm{~m} .4$ 个点的目标位置与幅度

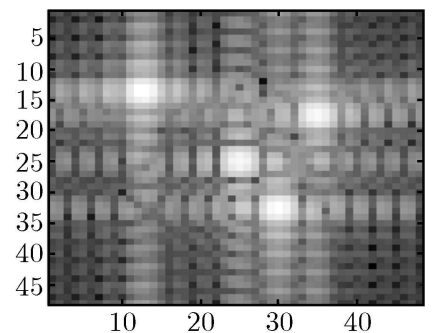

(a)

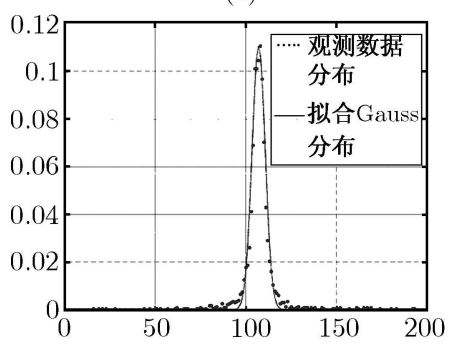

(c)

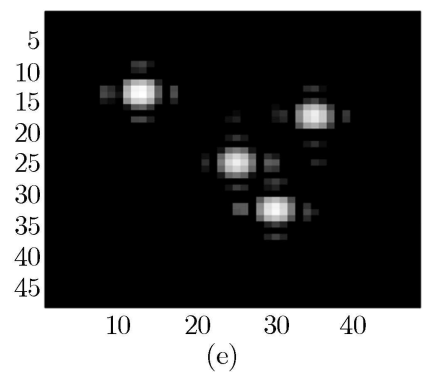

图 2 仿真复图像及去噪处理结果

(a) 不含噪分贝图; (b) 含噪分贝图; (c) 实部数据分布拟合图; (d) 文献 [6] 迭代方法 (分贝图); (e) 本文去噪结果 (分贝图); (f) 超分辨结果 (分贝图)
表 1 仿真图像目标位置与幅度参数

\begin{tabular}{cccccc}
\hline & 点目标 1 & 点目标 2 & 点目标 3 & 点目标 4 \\
\hline \multirow{2}{*}{ 位置 } & $x$ & 0 & 0.6 & -0.6 & -0.9 \\
\cline { 2 - 6 } & $y$ & 0 & 0.6 & 1.2 & 1.5 \\
\hline 幅度 & $6+9 \mathrm{j}$ & $7+10 \mathrm{j}$ & $8+8 \mathrm{j}$ & $10+9 \mathrm{j}$ \\
\hline
\end{tabular}

参数如表 1 所示: 在相位历史域进行三倍插值, 并添 加 $\sigma=0.1$ 的 Gauss 白噪声. 需要说明的是, 在仿真 过程中没有考虑加窗处理. 处理结果如图 2 所示.

说明:

(i) 由于 SAR 图像动态范围或过大, 或过小 (本 文实验用图像), 不便于显示, 为显示图像细节, 图 2 和 3 显示的是分贝图, 即 $f_{p}=20 \log _{10}\left(\left|f_{c}\right|\right), f_{c}$ 为复 图像数据.

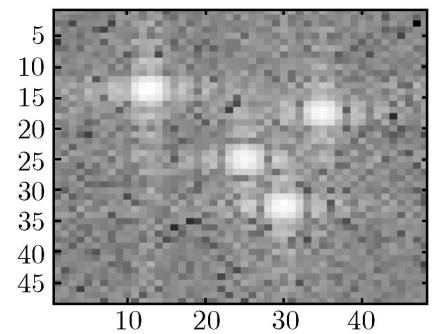

(b)

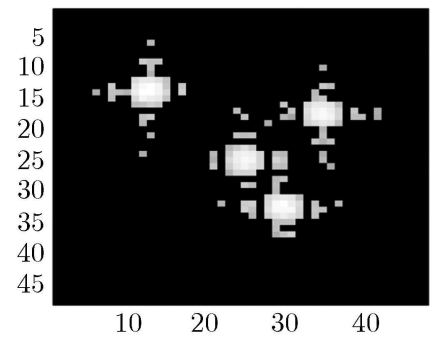

(d)

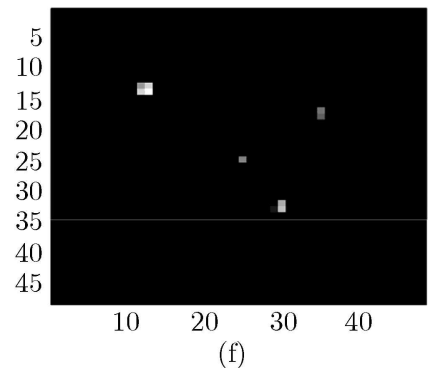

(f) 
表 2 仿真复图像处理结果定量指标评估结果

\begin{tabular}{ccccc}
\hline & 原图 & 文献 [6] 迭代结果 & 本文结果 & 超分辨结果 \\
\hline MSE & - & 0.0299 & 0.0035 & 0.0210 \\
峰值信噪比 $(\mathrm{dB})$ & - & 18.8352 & 27.6019 & 18.1845 \\
幅度最大值 & 1.4863 & 1.4659 & 1.4683 & 0 \\
幅度最小值 & 0 & 0 & 0.2525 & 0 \\
距离向分辨率 & 0.2651 & 0.2637 & 0.3861 & 0.0861 \\
(方位向分辨率) & 0.4110 & 0.4080 & $0 / 0$ & 0.1319 \\
等效视数 & 1.1044 & $0 / 0$ & 1 & $0 / 0$ \\
迭代次数 & - & 17 & 166 & 1 \\
每次迭代计算像素数 & - & $48 \times 48$ & 11 \\
\hline
\end{tabular}

(ii) 在表 2 等效视数 ENL 计算中,

$$
\mathrm{ENL}=\frac{\operatorname{mean}^{2} \text { (data) }}{\operatorname{std}^{2} \text { (data) }} .
$$

由于处理后图像均匀区域方差与均值均为 0 , 故在上 面表格中填写 $0 / 0$.

(iii) 在表 2 的分辨率计算中, 以中央点目标为例 进行计算. 其他 3 个点目标分辨率稍低. 2 个数值分 别为距离向、方位向分辨率.

(iv) 在图 2(c) 中的数据分布拟合中, 为统计方 便, 将原有数据范围经过线性变换到 $[0,200]$ 区间内. 且由于实部与虚部情况类似, 为节省篇幅, 省去虚部 拟合效果图.

(v) 在 MSE 计算中, 从图 2 看出, 超分辨效果 (f) 与原始图像 (a) 之间的差别比 (d) 的差别要大, 但是 由于数据值较小, 而且 MSE 反映一种综合差异, 故 (d) 与 (f) 差异并不明显.

(vi) 在迭代过程中, 当

$$
\frac{\left\|\boldsymbol{f}^{(n+1)}-\boldsymbol{f}^{(n)}\right\|_{2}^{2}}{\left\|\boldsymbol{f}^{(n+1)}\right\|_{2}^{2}} \leqslant \gamma
$$

时结束迭代过程. $\gamma$ 为一阈值, 可取 $10^{-6}$. 迭代次数 与第 2 节理论值相比要低. 分析其原因在于此处迭代 停止准则以整体误差较小为准则, 由于像素之间误差 的相互作用, 使得次数降低. 而且从理论上分析的只 是迭代最悲观结果. 而且从图 2 可看出, 迭代后误差 高于本文误差.

(vii) 图 2 中的超分辨结果是在本文去噪结果基 础上, 取 $\lambda k>2 \sigma_{g}^{2}$ 进行处理得到的.
(2) MSATR 复图像 (见图 3 及表 3).

说明:

(i) 由于实际中难以得到真实参考实测图像, 故 不再计算 MSE、峰值信噪比.

(ii) 图 3 中, (b) 相比 (c) 与 (d), 强散射点亮的 原因在于迭代求解后, 除少量原像素为 0 的数据迭 代为 0 外, 其他本应收玫到 0 的像素点迭代为 $10^{-16}$ 量级, 这是 Matlab 中的缺省容差, 取对数后与其它 散射点相差不大. 而本文处理结果大部分像素为 0 , 取对数为 $-\infty$, 从而造成显示上的错觉. 可通过添加 容差达到与图 3(c) 相似的显示效果.

(iii) 分辨率以图 3(e) 中所示参考点为例进行计 算.

(iv) 在图 3(d) 中杂波中的若干强散射点得以保 留, 这有利于后续的目标检测.

此外, 本文主要考虑强散射点目标, 在处理后图 像中, 阴影特征被去除. 这一不足可以通过在正则化 模型中添加边缘保持项, 或者在原图中利用文献 [17] 等的边缘检测与本文结果相叠加来弥补, 不在本文讨 论范围.

\section{6 结论}

SAR 图像去噪是提高 SAR 图像质量的一种重 要方式, 可以提高目标检测与识别等后续应用性能. 现有基于 $l_{k}$ 范数正则化模型的 SAR 图像去噪方法 存在求解需迭代、参数需人工确定的问题. 本文从参 数估计角度研究 SAR 复图像域上基于该模型的快速 


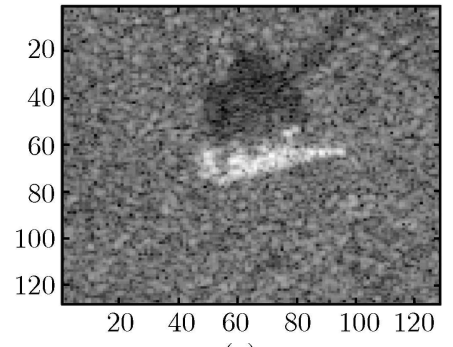

(a)

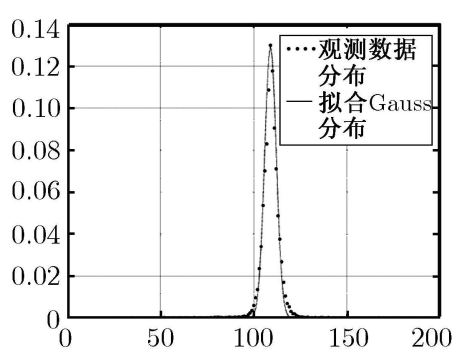

(b)

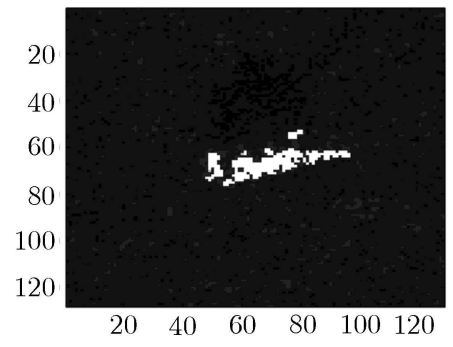

(c)

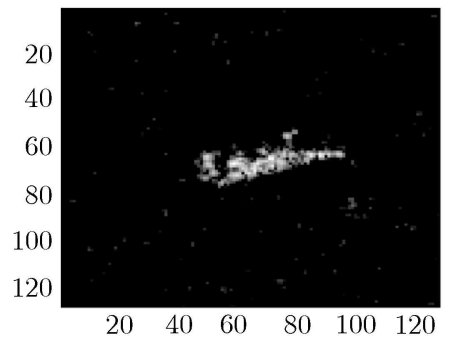

(d)

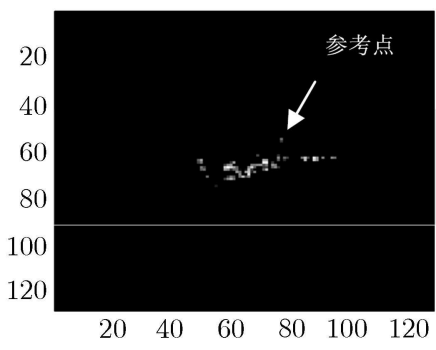

(e)

图 3 MSTAR 复图像 (HB03335.015) 及去噪处理结果

(a) 原始分贝图像; (b) 实部数据拟合图; (c) 文献 [6] 方法 (分贝图); (d) 本文方法 (分贝图); (e) 超分辨结果 (分贝图)

表 3 MSTAR 复图像处理定量指标评估结果

\begin{tabular}{ccccc}
\hline & 原图 & 文献 [6] 迭代结果 & 本文结果 & 超分辨结果 \\
\hline 幅度最大值 & 1.3460 & 1.3350 & 1.3432 & 1.2931 \\
幅度最小值 & 0 & 0 & 0 & 0 \\
距离向分辨率 & 0.2954 & 0.2836 & 0.2748 & 0.1833 \\
(方位向分辨率) & 0.2932 & 0.2746 & 0.2691 & 0.1744 \\
等效视数 & 0.8061 & $0 / 0$ & $0 / 0$ & 1 \\
迭代次数 & - & 17 & 633 & $0 / 0$ \\
每次迭代计算像素数 & - & $128 \times 128$ & & 94 \\
\hline
\end{tabular}

自适应去噪方法. 从现有参数选择方法的分析引出 研究模型的解析解的必要性. 通过最小化均方误差 以及单调有界序列相关原理, 得到了正则化参数的 选择方法. 以及模型的解析解. 从而大大加快了处理 速度, 并从理论上分析得到本文方法比迭代方法快
速的数值极限. 该分析过程理论上比现有方法更加 严谨. 最后, 建立了正则化参数与处理后 SAR 图像 分辨率的联系, 证明了基于 $l_{k}$ 范数的正则化模型可 以提高 SAR 图像点目标分辨率. 本文方法可扩展到 SAR 幅度图像域与功率图像域. 


\section{参考文献}

1 Li Z F, Bao Z, Yang F F. Ground moving target detection and location based on SAR images for distributed spaceborne SAR. Sci China Ser F-Inf Sci, 2005, 48(5): 632-646

2 Cetin M, Karl W C, Castanon D A. Feature enhancement and ATR performance using nonquadratic optimization-based SAR imaging. IEEE Trans Aerospace Electron Syst, 2003, 39(4): 1375-1395

3 王正明, 朱炬波. SAR 图像提高分辨率技术. 北京: 科学出版社, 2006

4 Cetin M, Karl W C. Feature-enhanced synthetic aperture radar image formation based on nonquadratic regularization. IEEE Trans Image Process, 2002, 10(4): 623-631

5 汪雄良, 王正明, 赵侠, 等. 基于 $l_{k}$ 范数正则化方法的 SAR 图像超分辨. 宇航学报, 2005, 26(增刊)：77-82

6 赵侠, 朱炬波, 王正明. SAR 复图像上的噪声抑制与目标特征提取. 电子学报, 2005, 33(12): 2135-2138

7 Achim A, Kuruoglu E E, Zerubia J. SAR image filtering based on the heavy-tailed Rayleigh model. IEEE Trans Image Process, 2006, 15(9): 2686-2693

8 周宏潮, 王正明, 基于稀疏型先验的光学及 SAR 图像分辨率增强的统一框架. 量子电子学报, 2006, 23(1)：135-140

9 Steven M Kay. 统计信号处理基础一一估计与检测理论. 罗鹏飞, 译. 北京: 电子工业出版社, 2003

10 Aster R, Borchers B, Thurder C. Parameter Estimation and Inverse Problems. Sandiego: Academic Press, 2004

11 Charbonnier P, Laure B, Aubert G, et al. Deterministic edge-preserving regularization in computed imaging. IEEE Trans Image Process, 1997, 6(2): 298-311

12 Gorman J D, Hero A O. Lower bounds for parametric estimation with constraints. IEEE Trans Inform Theor, 1990, 26(6): $1285-1301$

13 陈希獳, 王松桂. 近代回归分析一原理方法及应用. 合肥: 安徽教育出版社, 1987

14 游扬声, 王新洲, 刘星. 广义岭估计的直接解法. 武汉大学学报 (自然科学版), 2002, 27(2): 175-178

15 张澄波. 综合孔径雷达: 原理、系统分析与应用. 北京: 科学出版社, 1989

16 Michael J G, Lee C P, Inder J G, et al. A parametric model for synthetic aperture radar measurements. IEEE Trans Anten Propag, 1999, 47(7): 1179-1185

17 付琨, 匡纲要, 郁文贤.一种合成孔径雷达阴影与目标检测的方法. 软件学报, 2002, 13(4): 818-826 